\title{
Endocrine Therapy Toxicity
}

National Cancer Institute

\section{Source}

National Cancer Institute. Endocrine Therapy Toxicity. NCI Thesaurus. Code C138163.

Indicates that a toxicity adverse effect has been experienced during endocrine drug treatment. 